\title{
A Newtonian pre-introduction to gravitational lenses
}

\author{
T. Garel * \\ Service de Physique Théorique, CEA/DSM/SPhT \\ Unité de recherche associée au CNRS \\ 91191 Gif sur Yvette Cedex, France
}

August 20, 2018

\begin{abstract}
Understanding the deflection of light by a massive deflector, as well as the associated gravitational lens phenomena, require the use of the theory of General Relativity. I consider here a classical analogy, based on Newton's equation of motion for massive particles. These particles are emitted by a distant source and deflected by the gravitational field of a (opaque) star or of a (transparent) galaxy. The dependence of the deviation angle $D$ on the impact parameter $b$, and the - Euclidean - geometry of the (source, deflector, earth) triplet, imply that different particle trajectories may reach an earth based observer. Since $D(b)$ does not depend on the mass of the particles, a (Newtonian) flavor of gravitational lenses phenomena is naively obtained by setting the particles' velocity equal to the speed of light. Orders of magnitude are obtained through this classical approach, and are compared to the General Relativity results.
\end{abstract}

Saclay T03/171

*Member of CNRS 


\section{Introduction}

Gravitational lenses phenomena [1, 2, 3, 4, 5, 6] rest on the gravitational deflection of light, and their explanation require the use of General Relativity (the gravitational deflection of light by the sun, of order $10^{-5} \mathrm{rd}$, was indeed a major issue at the very beginning of the theory). This theory uses a rather heavy technical machinery, which is not easily accessible to undergraduate students. One of the motivations of the present paper is to offer, through a classical approach, a feeling (and orders of magnitude) for gravitational lenses phenomena.

Apart from extreme cases (neutron stars, black holes,...), the gravitational defection of light in General Relativity (GR) is small, and can be described by a weak gravity approximation [1, 2, 3. For quasistationary isolated mass distributions, the evolution of the unit tangent vector $(\vec{e}(s))$ to a light ray $(\vec{r}(s))$ is given to lowest order (see below) by

$$
\frac{d \vec{e}(s)}{d s}=-\frac{2}{c^{2}} \vec{\nabla}_{\perp} U
$$

where $c$ is the speed of light, $U$ the gravitational potential created by static mass distributions, and $\left(\vec{\nabla}_{\perp} U=\vec{\nabla} U-\vec{e}(\vec{e} \cdot \vec{\nabla} U)\right)$. The weak gravity approximation corresponds to $\frac{U}{c^{2}}<<1$, and "lowest order" means that the typical velocity $v$ of the mass distribution is small compared to $c$ : A more rigorous calculation indeed shows that there appear other terms in the r.h.s of equation (11), among which the lowest order term is of order $\mathrm{O}\left(\frac{v}{c}\right)$ ) (see eq. 4.17, p. 124 of [2]).

Equation (11) bears some resemblance to a classical equation of motion; it is indeed a familiar remark that Newton's classical equation for a massive particle in the gravitational field of a deflector leads to a mass independent deflection angle (identity of inertial and gravitational masses). In this paper, we study the classical mechanical problem defined by the triplet (particle source $(\mathrm{S})$, deflector $(\Delta)$, earth based observer $(\mathrm{E}))$. An important ingredient of this study is the dependence of the gravitational deflection angle $D$ on the impact parameter $b$ of the particles. Since the (Euclidean) distances between $(\mathrm{S}),(\Delta)$ and $(\mathrm{E})$ are finite, the exact calculation of $D(b)$ is rather involved, even if one takes advantage of the central character of the gravitational force and of some invariant properties (we use here the Runge-Lenz vector and tensor). A rather quantitative approach is used here to derive the main 
features of the deflection $D(b)$ for the case of spherical deflectors (mass $M$, radius $R$ ).

To make contact with the (GR) weak gravity approximation, we consider a similar approximation for the classical mechanical study, namely we consider the limit (Gravitational energy $<<$ Kinetic energy). Further, if $\left(v_{\infty}\right)$ is a measure of the particle velocity as it leaves the source, one has at some point to make the (delicate) correspondence $v_{\infty} \rightarrow c$ in the classical mechanical problem. In this paper we follow this view of the gravitational deflection of light to obtain orders of magnitude, using typical undergraduate skills. It is shown that, in some cases, several particle trajectories may reach observer (E). These trajectories translate into a basic gravitational lensing effect: the source (S) may give multiple (photonic) signals for observer (E). The time delay between the reception of these different trajectories (or signals) is also considered.

The plan of the paper is as follows. The gravitational action of the deflector, such as the sun (opaque star) or the Milky Way (transparent galaxy), is studied in Section 2. For simplicity, we assume there that the source (S) and the observer (E) are both at infinity. When (S) and (E) are at a finite distance from the deflector, a little bit of geometry shows that that (S) may have multiple "images" (Section [3). Finally, an estimate of the time lag between the reception of these "images" is obtained, via the analogy of particle mechanics and geometrical optics (Section 4).

\section{Study of the classical mechanical deflection problem}

We consider a source $(\mathrm{S})$ which emits, in an isotropic fashion, isovelocity particles $\left(|\vec{v}|=v_{\infty}\right)$ of mass $m$. Unless otherwise stated, we will consider a point source. We focus our interest on the deflector and take the source and the observer at infinity. Let $\vec{v}_{\infty}=v_{\infty} \vec{u}_{i}$ be the initial velocity of a test particle. The deflector $(\Delta)$ may be either opaque or transparent to the particles. The former case will be illustrated by the sun (radius $R_{\odot} \simeq 7 \cdot 10^{8} \mathrm{~m}$ and mass $M_{\odot} \simeq 2 \cdot 10^{30} \mathrm{~kg}$ ), and the latter by a spherical version of the Milky 
Way ${ }^{1}$ (radius $R_{G} \simeq 2 \cdot 10^{4}$ light years $(\text { ly })^{2}$ and mass $\left.M_{G} \simeq 10^{12} M_{\odot}\right)$.

\subsection{The opaque detector}

The impact parameter $b$ and deviation angle $D$ are defined in Figure 1. The study of the classical Kepler problem for $b>R^{3}$ can be found in standard textbooks (see e.g. [7]). If $\left(\vec{u}_{i}\right)$ and $\left(\vec{u}_{f}\right)$ are the unit vectors along initial and final velocities, we have $\cos D(b)=\vec{u}_{i} \cdot \vec{u}_{f}$.

The Runge-Lenz vector of the Kepler problem reads

$$
\vec{A}=\vec{v} \times \vec{L}-\mathcal{G} M m \frac{\vec{r}}{r}
$$

where $\vec{r}$ is the particule position measured from the center of the deflector $(\Delta), \vec{L}=\vec{r} \times m \vec{v}$ its angular momentum, and $\mathcal{G}$ the universal gravity constant. As is easily checked, $\vec{A}$ is an invariant vector of the motion and writing $\overrightarrow{A_{i}}=\overrightarrow{A_{f}}$ [8] leads to

$$
\tan \frac{D}{2}=\frac{\mathcal{G} M}{b v_{\infty}^{2}}
$$

The weak gravity condition means that the potential energy $m U(b)$ is a small part of the total energy, that is $\frac{\mathcal{G} M}{b v_{\infty}^{2}}<<1$. The deviation is then given by

$$
D(b) \simeq \frac{2 \mathcal{G} M}{b v_{\infty}^{2}}
$$

The (GR) result is obtained from equation (11) as $D_{G R}(b) \sim \frac{4 \mathcal{G} M}{b c^{2}}$. Applying these results to the sun at grazing incidence yields $D_{G R}\left(R_{\odot}\right) \sim 810^{-6}$ rd.

Two remarks are in order (i) The naive identification $\left(v_{\infty} \rightarrow c\right)$, yields an expression which is half the exact (GR) result (ii) In classical mechanics, energy conservation implies that $v^{2}(r)=v_{\infty}^{2}+\frac{2 \mathcal{G} M}{r}$. If one identifies $v_{\infty}$ with

\footnotetext{
${ }^{1}$ see e.g.

http://hypertextbook.com/facts/2000/AlinaVayntrub.shtml

http://www.stdimension.de/int/Cartography/mwtour.htm

${ }^{2}$ One light year represents a distance of approximately $9.5 \cdot 10^{15} \mathrm{~m}$.

${ }^{3}$ We do not make the distinction between the impact parameter $(b)$ and the minimum distance approach $\left(r_{0}\right)$. The exact relation $r_{0}=b\left(\frac{1-\sin \frac{D}{2}}{\cos \frac{D}{2}}\right)$ shows that for small $D$, one has $r_{0} \simeq b$.
} 
$c$, the velocity $v(r)$ is greater than $c$, the (weak) correction term being of order $\frac{\mathcal{G} M}{r c^{2}}$. For small $D$, the minimum distance $r_{0}$ approach is of order $b$, leading to a maximum supraluminal correction for the velocity of order $\frac{\mathcal{G} M}{b c^{2}}$, that is small. As a temporary conclusion, I would say that for practical purposes, the weak gravity limit of the classical case, together with the identification $v_{\infty} \rightarrow c$, yields a correct order of magnitude for the (GR) value. Some inconsistencies of classical mechanics appear in this identification, but are small in the weak gravity limit.

\subsection{The transparent deflector}

A preliminary remark is that $D(b)$ is not a monotonous function, since it vanishes both for $b=0$ and $b=\infty$. For $(b>R)$, the deviation is exactly given by equation (3), the weak gravity approximation corresponding to equation (41).

The case $(b<R)$ requires the study of two distinct phases of the motion, since the gravitational field $\vec{g}(\vec{r})$ on the test particle has different expressions, depending on the particle position $\vec{r}$. We respectively denote these phases by (out) and (in). Their respective contribution to the total deviation $D(b)$ will be denoted by $D_{(\text {out })}$ and $D_{(i n)}$. We further assume that the deflecting galaxy is homogeneous, and neglect all collisions with the particles in phase (in).

In phase (in) of the motion, Gauss' theorem gives

$$
\ddot{\vec{r}}=\vec{g}_{(i n)}(\vec{r})=-\frac{\mathcal{G} M}{R^{3}} \vec{r}=-\omega_{0}^{2} \vec{r}
$$

This harmonic motion has period $T_{0}=\frac{2 \pi}{\omega_{0}}=2 \pi \sqrt{\frac{R^{3}}{\mathcal{G M}}}$. The trajectory inside the deflector is elliptic or partially elliptic. One should express the initial and final boundary conditions to get the deflection $D_{(i n)}(b)$. These boundary conditions depend on phase (out) of the trajectory $(r>R)$, where Newton's equation reads

$$
\ddot{\vec{r}}=\vec{g}_{(\text {out })}(\vec{r})=-\frac{\mathcal{G} M}{r^{3}} \vec{r}
$$

The total deviation $D(b)$ is given by

$$
D(b)=D_{(\text {in })}(b)+D_{(\text {out })}(b)
$$




\subsubsection{Phase (out): $(r>R)$}

\section{(a) Rigorous solution}

We want to calculate the deviation between initial (source $\mathrm{S}$ ) and final (entry into deflector $(\Delta)$ ) points. The final point $\mathrm{M}$ has position $\vec{r}_{i n}=\overrightarrow{O M}$ and velocity $\vec{v}_{i n}$ (Figure $2(\mathrm{a})$ ), with $\left|\vec{r}_{i n}\right|=R$, and $\vec{v}_{i n}=v_{0} \vec{u}_{i n}$. Energy conservation yields $v_{0}^{2}=v_{\infty}^{2}+2 \frac{\mathcal{G} M}{R}$. The deviation $D_{\text {out }}^{(1)}$ for this part of the (out) trajectory is given by $\cos D_{\text {out }}^{(1)}=\vec{u}_{i} \cdot \vec{u}_{i n}$.

Equating the projections of the Runge-Lenz vector (eq. (2)) along $\vec{u}_{i}$ for the initial $(\mathrm{S})$ and final $(\mathrm{M})$ points leads to

$$
\mathcal{G} M m=\vec{u}_{i} \cdot\left(\vec{v}_{i n} \times \vec{L}-\mathcal{G} M m \frac{\vec{r}_{i n}}{R}\right)
$$

Defining $\cos (\pi-\Phi)=\vec{u}_{i} \cdot \frac{\vec{r}_{i n}}{R}$ (Figure 2(a)), we get

$$
\sin D_{\text {out }}^{(1)}=\frac{\mathcal{G} M}{b v_{0} v_{\infty}}(1-\cos \Phi)
$$

The exact solution of the Kepler problem (see eq (14,7), p.46 of [7]) yields

$$
\Phi=-\arccos \left(\frac{\frac{b}{R}-d}{\sqrt{1+d^{2}}}\right)+\arccos \left(\frac{-d}{\sqrt{1+d^{2}}}\right)
$$

with $d=\frac{\mathcal{G} M}{b v_{\infty}^{2}}$.

Taking into account the symmetrical $(\Delta E)$ contribution (deviation $D_{\text {out }}^{(2)}$ ) finally gives

$$
D_{\text {out }}(b)=2 \arcsin \left(\frac{\mathcal{G} M}{b v_{0} v_{\infty}}(1-\cos \Phi)\right)
$$

where $\Phi$ is given by (10).

\section{(b) Weak gravity approximation}

As previously stated, we expect gravitational deviations to be weak. Equation (11) shows that the weak gravity ("small $\mathcal{G}$ ") limit can be obtained by setting $d=0$ in eq. (10). The weak gravity approximation therefore reads

$$
D_{\text {out }}(b) \simeq 2 \frac{\mathcal{G} M}{b v_{\infty}^{2}}\left(1-\sqrt{1-\frac{b^{2}}{R^{2}}}\right)
$$

In particular, the small $b$ limit is given by $D_{\text {out }}(b) \sim \frac{b}{f}$, with $f=\frac{R^{2} v_{\infty}^{2}}{\mathcal{G} M}$. 


\subsubsection{Phase (in): $(r<R)$}

\section{(a) Rigorous solution}

The geometry of the (in) phase is shown in Figure 2 (b). The angle $\alpha$ is the angle between the radius vector $\vec{r}_{i n}$ and the velocity $\vec{v}_{i n}$ as the particle enters the deflector (Figure 2(a)). From the conservation of the angular momentum, one has

$$
m v_{\infty} b=m v_{0} R \sin \alpha
$$

where $v_{0}^{2}=v_{\infty}^{2}+2 \frac{\mathcal{G} M}{R}$.

To calculate the deviation $D_{i n}(b)$ between the entry and exit points, one may solve the harmonic motion of eq. (5). A more convenient way is to use the Runge-Lenz matrix invariant associated with the harmonic oscillator

$$
\mathbf{A}=\frac{m}{2}\left(\omega_{0}^{2} \mathbf{r r}+\mathbf{v} \mathbf{v}\right)
$$

Starting from the invariance of $\mathbf{A}$, simple calculations 9 show that

$$
D_{(i n)}(b)=2(\alpha-\beta)
$$

where $\sin \alpha=\frac{b v_{\infty}}{R v_{0}}$, and $\tan 2 \beta=\frac{v_{0}^{2} \sin 2 \alpha}{\omega_{0}^{2} R^{2}+v_{0}^{2} \cos 2 \alpha}$.

\section{(b) Weak gravity approximation}

In this approximation, we obtain $\sin \alpha \simeq \frac{b}{R}$, and

$$
D_{i n}(b) \simeq \tan D_{i n}(b)=\tan 2(\alpha-\beta) \simeq \frac{\mathcal{G} M}{R v_{\infty}^{2}} \sin 2 \alpha
$$

\subsubsection{Conclusion on the transparent deflector}

The total deviation $D(b)$, for $b<R$ and in the weak gravity approximation, is given by eq. (12) and (16). Setting $u=\frac{b}{R}$, we have

$$
D(b)=D_{(\text {in })}(b)+D_{(\text {out })}(b)=2 \frac{\mathcal{G} M}{R v_{\infty}^{2}}\left(\frac{1-\left(1-u^{2}\right)^{\frac{3}{2}}}{u}\right)
$$

When $v_{\infty} \rightarrow c$, this result can be compared to the (GR) result [4, 5]. Notwithstanding the (ubiquitous) factor of 2 between classical mechanics 
and (GR), eq. (17) is in agreement with eq. (7) of reference [5]. Note that equation (17) can be also be written in a way similar to eq. (41), namely

$$
D(b)=2 \frac{\mathcal{G} M(b)}{b v_{\infty}^{2}}
$$

where $M(b)=M\left(1-\left(1-\frac{b^{2}}{R^{2}}\right)^{\frac{3}{2}}\right)$ is the partial deflector mass contained in a cylinder of radius $b$.

For small $b$, one gets $D(b) \sim 3 \frac{b}{f}$. The length $f=\frac{R^{2} v_{\infty}^{2}}{\mathcal{G} M}$ can be viewed as a focal length; its order of magnitude for our model galaxy is $2.510^{9} \mathrm{ly}$, much bigger than $R_{G} \sim 210^{4}$ ly. Note also that $D(b)$ has a maximum for $b \simeq 0.93 R_{G}$, with $D_{\max } \simeq 1.610^{-5} \mathrm{rd}$.

Using the results of equations (4),(17), we show in Figure 3 the deviation $D(b)$ as a function of the impact parameter $b$, for opaque and transparent deflectors.

\section{$3 \quad$ Finite distance geometry and multiple tra- jectories}

We now use our results to discuss an experimentally more relevant situation, where both the source $(\mathrm{S})$ and the observer (E) are at a finite distance from the deflector $(\Delta)$. The - Euclidean - geometry is shown in Figure 4. We have $r_{S}=S_{0} \Delta=S H, r_{E}=\Delta E$. Given the previous orders of magnitude, the angles such as $\beta=\widehat{S E} \Delta, \theta=\widehat{\triangle E Y}$ and the deviation $D$, are assumed to be small. This implies in particular that $b=\Delta Y=r_{E} \tan \theta<<r_{E}, r_{S}$. We have from (Euclidean) geometry

$$
\frac{\sin \widehat{S E Y}}{S Y}=\frac{\sin \widehat{E S Y}}{E Y}
$$

From triangle SHY, one has

$$
S Y^{2}=S H^{2}+H Y^{2}=r_{S}^{2}+\left(r_{E} \tan \theta-\left(r_{E}+r_{S}\right) \tan \beta\right)^{2}
$$

yielding for small angles, $S Y \simeq r_{S}\left(1+O\left(\theta^{2}, \beta^{2}, \theta \beta\right)\right)$.

From triangle $Y E \Delta$ one has

$$
E Y^{2}=r_{E}^{2}+\Delta Y^{2}=r_{E}^{2}\left(1+\tan ^{2} \theta\right)
$$


yielding for small angles, $E Y \simeq r_{E}\left(1+O\left(\theta^{2}\right)\right)$.

Plugging these values in eq. (19), we get to lowest order in the angles $\theta, \beta, .$.

$$
\frac{\widehat{S E Y}}{r_{S}} \simeq \frac{\widehat{E S Y}}{r_{E}} \simeq \frac{\widehat{S E Y}+\widehat{E S Y}}{r_{S}+r_{E}} \simeq \frac{D}{r_{S}+r_{E}}
$$

Since $\widehat{S E Y}=\theta-\beta \simeq \frac{b}{r_{E}}-\beta$, we finally obtain

$$
D(b) \simeq \frac{r_{S}+r_{E}}{r_{S}}\left(\frac{b}{r_{E}}-\beta\right)
$$

Equation (23), which expresses the condition that a particle emitted from (S) reaches the earth, is represented by the dotted lines in Figure 3. For an opaque deflector, one may get one or two solutions for $b$. For a transparent deflector, one may get up to three solutions for $b$. Rather than studying the full problem as a function of $\beta, r_{S}, r_{E}, \ldots$, we illustrate some particular situations

\subsection{A generic case}

This case corresponds to a non zero $\beta$ angle (Figure 4 ). We focus our interest on points (1) and (2) of Figure 3, which are the intersections of the geometrical equation

$$
D(b) \sim \frac{r_{S}+r_{E}}{r_{S}}\left(\frac{b}{r_{E}}-\beta\right)
$$

with the $b>R$ gravitational deflection $D(b)$ of Section 2 (see Figure 3). Since we have $D(b) \sim 2 \frac{\mathcal{G} M}{b v_{\infty}^{2}}$, setting $\theta=\frac{b}{r_{E}}$ leads to

$$
\theta^{2}-\beta \theta-\theta_{E}^{2}=0
$$

where

$$
\theta_{E}^{2} \sim 2 \frac{\mathcal{G} M}{v_{\infty}^{2}} \frac{r_{S}}{r_{E}\left(r_{S}+r_{E}\right)}
$$

In this case, there are two trajectories in the $S \Delta E$ plane that reach (E). In photon language, the observer sees two images $(S 1)$ and $(S 2)$ of the source $(\mathrm{S})$, on opposite sides of the deflector, with

$$
\theta_{1,2}=\frac{\beta \pm \sqrt{\beta^{2}+4 \theta_{E}^{2}}}{2}=\frac{b_{1,2}}{r_{E}}
$$

For a transparent deflector, one has another image of the source, corresponding to the point labeled (3) in Figure 3(b). 


\subsection{Einstein rings}

This case corresponds to the alignment of $(\mathrm{S}),(\Delta)$ and $(\mathrm{E})(\beta=0)$. Due to the symmetry of revolution around the $S \Delta E$ axis, all trajectories on the angular cone $\theta=\theta_{E}=\frac{b}{r_{E}}$ reach the earth. In photon language, this means that the observer sees a ring image of the point source S. For a transparent deflector, one also has a direct image. For our model galaxy and $r_{E} \sim r_{S} \sim$ $10^{9} \mathrm{ly}$, a typical value is $\theta_{E} \sim 10^{-5} \mathrm{rd}$.

\subsection{The case of a moving deflector}

We briefly consider this case (called microlensing), because of its experimental relevance. Since a detailed comparison with the experiments require the use of General Relativity [1, 2, 3, we limit our presentation to orders of magnitude calculations. If the deflector $(\Delta)$ moves, with a velocity $v_{\Delta}$, in a direction perpendicular to the (SE) axis (Figure 4), the above calculations suggest the following scenario: for $\beta=0$ (ring image), there is a sudden increase in the signal received by the observer, since two trajectories only survive for $\beta \neq 0$. Physically the transition is gradual, and the observer will receive a gravitationally enhanced signal when the position of deflector $(\Delta)$ is within a distance $b_{E} \sim r_{E} \theta_{E}$ from the full alignment position of the previous section ${ }^{4}$ The corresponding time interval is $t_{E} \sim \frac{b_{E}}{v_{\Delta}}$. For distant sources $\left(r_{S}>>r_{E}\right)$, an experimental situation corresponding to a sun-like deflector, with $v_{\Delta} \sim 200 \mathrm{~km} \mathrm{~s}^{-1}, r_{E} \sim 3.610^{4}$ ly and $v_{\infty}=c$, yields an enhanced signal during an interval $t_{E} \sim 10^{6}-10^{7} \mathrm{~s}$, of order one month.

\section{Time lags}

\subsection{A simple optical analogy}

We have seen that several trajectories- or light rays- may reach (E) because of the gravitational deflection. Can one further extend the mechanical-optical analogy by finding the time lag between the reception of these trajectories -or light rays- ?

\footnotetext{
${ }^{4}$ A simple way to calculate the amplification factor is to use the non linear relation $\theta(\beta)$ of equation (27). The flux emitted by an extended source is proportional to $\beta d \beta$, and the flux received by the observer is proportional to $\theta d \theta$. The total amplification factor is given by $\mathcal{A}=\left|\frac{\theta d \theta}{\beta d \beta}\right|_{1}+\left|\frac{\theta d \theta}{\beta d \beta}\right|_{2}$.
} 
We first consider the transparent deflector for $b<<R$, where we found $D(b) \sim 3 \frac{b}{f}$ in section 2. This result may be compared with the optical deviation of a spherical glass lens, of radius $\rho$ and of optical index $\nu$ which reads

$$
D_{\text {opt }}=2 \frac{\nu-1}{\nu} \frac{b}{\rho}=\frac{b}{f_{\text {opt }}}
$$

where $b$ is the impact parameter of the light ray and $f_{\text {opt }}=\frac{\nu \rho}{2(\nu-1)}$ is the focal distance of the lens. The comparison of the gravitational and optical deviations suggests that the gravitational deviation may be understood through a gravitational index $n_{\text {grav }}$, with $n_{\text {grav }} \neq 1$.

\subsection{Particule trajectories and geometrical optics}

The preceding remark can be extended and formalized as follows. Energy conservation for a central potential $U(r)$ reads, in usual polar coordinates

$$
E=\frac{m}{2} \vec{v}^{2}+m U(r)=\frac{m}{2}\left(\dot{r}^{2}+r^{2} \dot{\theta}^{2}\right)+m U(r)
$$

Defining $\tan \Psi(r)=\frac{r(\theta)}{r^{\prime}(\theta)}$, we have

$$
E=\frac{\vec{L}^{2}}{2 m r^{2} \sin ^{2} \Psi(r)}+m U(r)
$$

which can be rewritten as

$$
n_{U}(r) r \sin \Psi(r)=\left(\frac{\vec{L}^{2}}{2 m E}\right)^{\frac{1}{2}}
$$

where the "index" $n_{U}(r)$, associated to the potential $U(r)$ is given by $n_{U}(r)=$ $\sqrt{1-\frac{m U(r)}{E}}$. Equation (31) is analogous to Bouguer's relation for the propagation of light rays in a spherically symmetric medium of index $n_{U}(r)$ [10].

We will illustrate this analogy with the case $b>R$, where $U(r)=-\frac{\mathcal{G} M}{r}$. The associated gravitational index reads

$$
n_{\text {grav }}(r)=\sqrt{1+\frac{2 \mathcal{G} M}{v_{\infty}^{2} r}} \simeq 1+\frac{\mathcal{G} M}{v_{\infty}^{2} r}
$$

where we have used the weak gravity approximation. Defining an analog $L_{\text {grav }}$ of the optical path, we may express the time lag $\delta t_{12}$ between the reception on earth of trajectories (1) and (2) of section 3.1 as 


$$
\delta t_{12}=\frac{L_{2}-L_{1}}{v_{\infty}}=\frac{\int_{(2)} n_{\text {grav }}(r) d s_{2}-\int_{(1)} n_{\text {grav }}(r) d s_{1}}{v_{\infty}}
$$

From eqs. (32) and (33), one finds that $\delta t_{12}$ is the sum of a geometrical part $\delta t_{\text {geom }}=\frac{\int_{(2)} d s_{2}-\int_{(1)} d s_{1}}{v_{\infty}}$ and of a gravitational part

$$
\delta t_{\text {grav }}=\frac{\mathcal{G} M}{v_{\infty}^{3}}\left(\int_{(2)} \frac{d s_{2}}{r}-\int_{(1)} \frac{d s_{1}}{r}\right)
$$

The full calculation of the integrals in (34) (see eq. 8.30, p.240 of [2]), yields a result that depends only logarithmically on the geometrical parameters $\left(\beta, r_{S}, \ldots\right)$. We therefore estimate $\delta t_{\text {grav }} \sim \frac{\mathcal{G} M}{v_{\infty}^{3}}$, up to a numerical factor of order one. Setting $v_{\infty}=c$, we find $\delta t_{\text {grav }} \sim 10^{-5} \mathrm{~s}$ for the sun, and $\delta t_{\text {grav }} \sim 10^{7} \mathrm{~s}$ for our model galaxy. Experiments that confirm the double reception of the same "signal", with a gravitational time lag of order several months, can be found in references [1, 2, 3, 5, 6].

Finally, it of interest to note that a Fermat approach to the weak gravity approximation of (GR) yields an equation similar to equation (32), with a (GR) index $n_{G R} \sim 1+2 \frac{\mathcal{G} M}{c^{2} r}$ [11, 12].

\section{Conclusion}

We have studied, at a qualitative level, a classical mechanical introduction to gravitational lens phenomena. This approach rests on the fact that the gravitational deflection of a massive particle by a deflector is independent of the particle mass. It is only an approximation to the theory of General Relativity [11, 12, but I believe that this " $\vec{F}=m \vec{a}$ optics " [13, 14] brings together in a very pedagogical way problems of different origins. In particular, we have derived orders of magnitude for the weak gravity case, that can be compared -up to a factor 2- to the correct (GR) results. As a caveat, we have nevertheless pointed out that the particle velocity may become (weakly) supraluminal, and this (weak) inconsistency with relativity is to be kept in mind.

Beside the study of General Relativity, the interested student can carry further the present approach in several ways. I will only quote here the modeling of gravitational lenses by optical lenses of the appropriate shape 
[5], or the link between equations (5) and (6) stemming from conformal transformations [15].

It is a pleasure to thank F. Bernardeau for discussions. 


\section{References}

[1] C. Misner, K. Thorne and J. Wheeler, Gravitation (Freeman, San Francisco 1973).

[2] P. Schneider, J. Ehlers and E.E. Falco, Gravitational Lenses, (Springer Verlag, Berlin-New York, 1992).

[3] A.F. Zakharov and M.V. Sazhin, "Gravitational microlensing", Phys. Usp., 41, 945-982 (1998).

[4] R.R. Bourassa and R. Kantowski, "The theory of transparent garvitational lenses", Ap. J. 195, 13-21 (1975).

[5] R.J. Adler, W.C. Barber and M.E. Redar, "Gravitational lenses and plastic simulators", Am. J. Phys., 63, 536-541 (1995) and references therein.

[6] J. Wambsganss, "Gravity's kaleidoscope", Sci. Am., 285, 52-59 (2001).

[7] L. Landau and E.M. Lifchitz, Mécanique, Third Edition, Editions Mir, Moscou (1969).

[8] L. Basano and A. Bianchi, "Rutherford scattering via the Runge Lenz vector", Am. J. Phys., 48, 400-01 (1980).

[9] J. Sivardière, "Comments on the dynamical invariants of the Kepler and harmonic motions", Eur. J. Phys., 13, 64-69 (1992) and references therein.

[10] M. Born and E. Wolf, "Principles of optics"(Fourth edition), Pergamon Press, Oxford (1970), eq (7), chapter 3.2.1, page 123.

[11] M.J. Nandor and T.M. Helliwell, "Fermat's principle and multiple imaging by gravitational lenses", Am. J. Phys., 64, 45-49 (1996) and references therein.

[12] P.M. Alsing, "The optical-mechanical analogy for stationary metrics in general relativity", Am. J. Phys., 66, 779-790 (1998) and references therein. 
[13] J. Evans and M. Rosenquist, " $\vec{F}=m \vec{a}$ optics", Am. J. Phys., 54, 876883 (1986) and references therein.

[14] C. Bellver-Cebreros and M. Rodriguez-Danta, "Eikonal equation from continuum mechanics and analogy between equilibrium of a string and geometrical light rays", Am. J. Phys., 69, 360-367 (2001) and references therein.

[15] L. Mittag and M.J. Stephen, "Conformal transformations and the application of complex variables in mechanics and quantum mechanics", Am. J. Phys., 60, 207-11 (1992). 


\section{Figure Captions}

Figure 1: The deflection geometry for $b>R$, with $(\mathrm{S})$ and $(\mathrm{E})$ at infinity. The vectors $\vec{u}_{i}$ and $\vec{u}_{f}$ are the unit vectors of the initial (emission) and final (reception) directions. The deviation $D=\arccos \left(\vec{u}_{i} \cdot \vec{u}_{f}\right)$ is a function of the impact parameter $b$. The minimum distance approach is $r_{0}$, and $r_{0} \simeq b$ for small $D$.

Figure 2: The deflection geometry for the transparent deflector

(a) phase (out) for $b<R$. The vector $\vec{u}_{i n}$ is the unit vector along the velocity as the particule enters the deflector at point $\mathrm{M}\left(\vec{v}_{i n}=v_{0} \vec{u}_{i n}\right)$. The corresponding deviation is given by $D_{\text {out }}^{(1)}=\arccos \left(\vec{u}_{i} \cdot \vec{u}_{\text {in }}\right)$

(b) phase (in) for $b<R$. The particle enters deflector $(\Delta)$ at point $\mathrm{M}$, and exits at point N. The OX and OY axes are the eigenvectors of the Runge-Lenz tensor A. Points $\mathrm{M}$ and $\mathrm{N}$ are symmetric w.r.t. OY. For clarity purposes, the direction of $\vec{u}_{\text {in }}$ has been rotated with respect to Figure 2(a).

Figure 3: The qualitative variation of $D(b)$ (a) opaque deflector (b) transparent deflector (note the maximum for $b \sim R_{G}$ ). The dotted lines represent various cases of equation (23). Solutions (1) and (2) correspond to trajectories (1) and (2) of Figure 4.

Figure 4: A typical geometry for finite distance gravitational deflection. Trajectories (1) and (2), deflected by $(\Delta)$, reach the earth $(\mathrm{E})$ : the source (S) has two images (S1) and (S2). For a transparent deflector, one has a third image of the source ( see point (3) in Figure 3(b)), corresponding to a trajectory entering $(\Delta)$. 
(S)

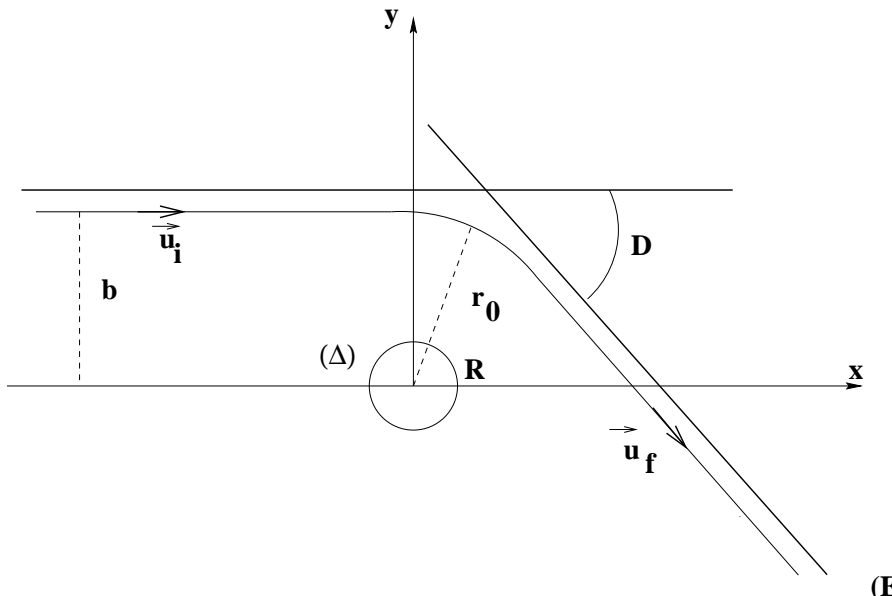

(E)

Figure 1

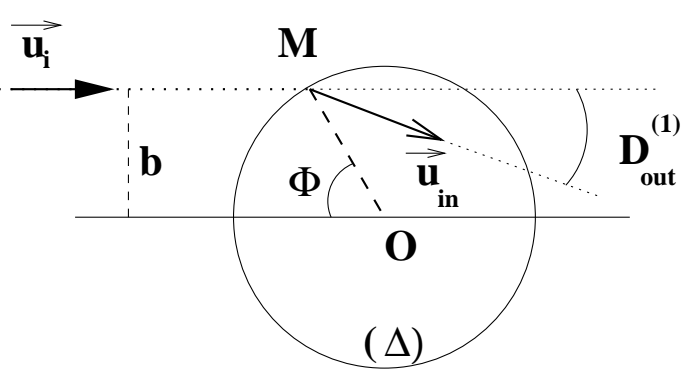

(a)

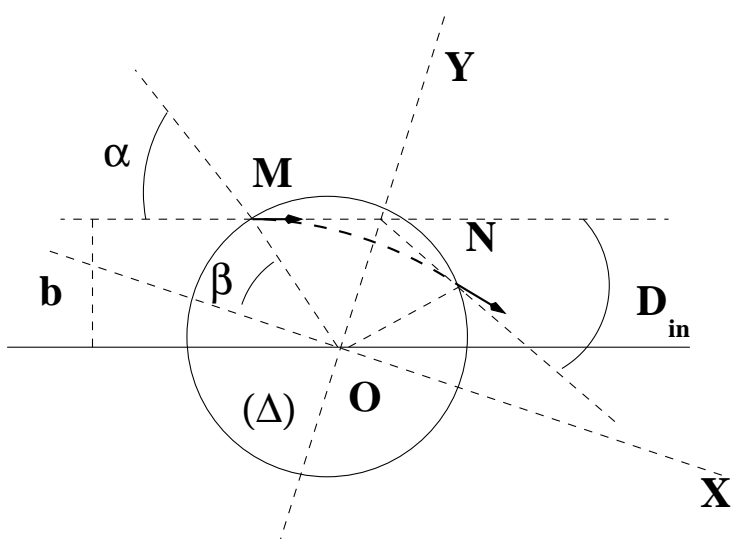

(b)

Figure 2 


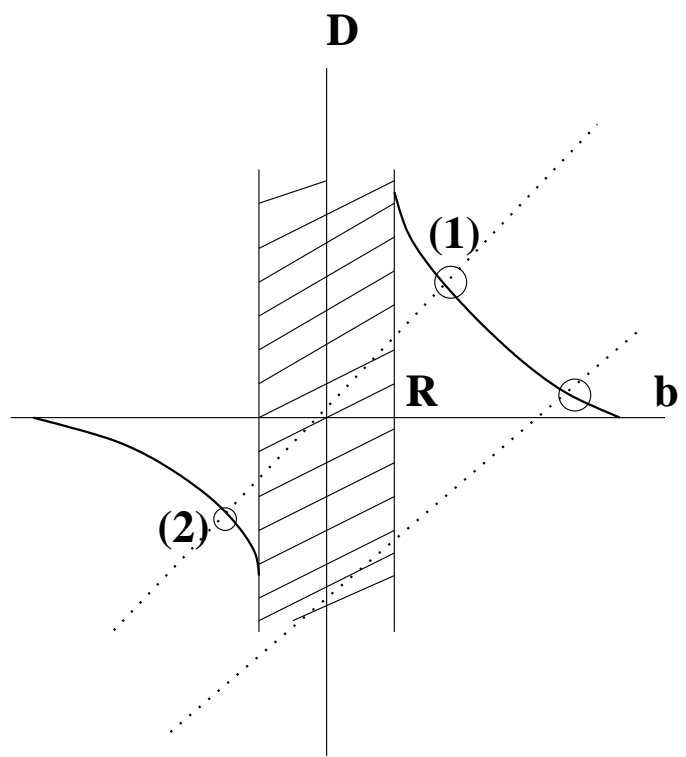

(a)

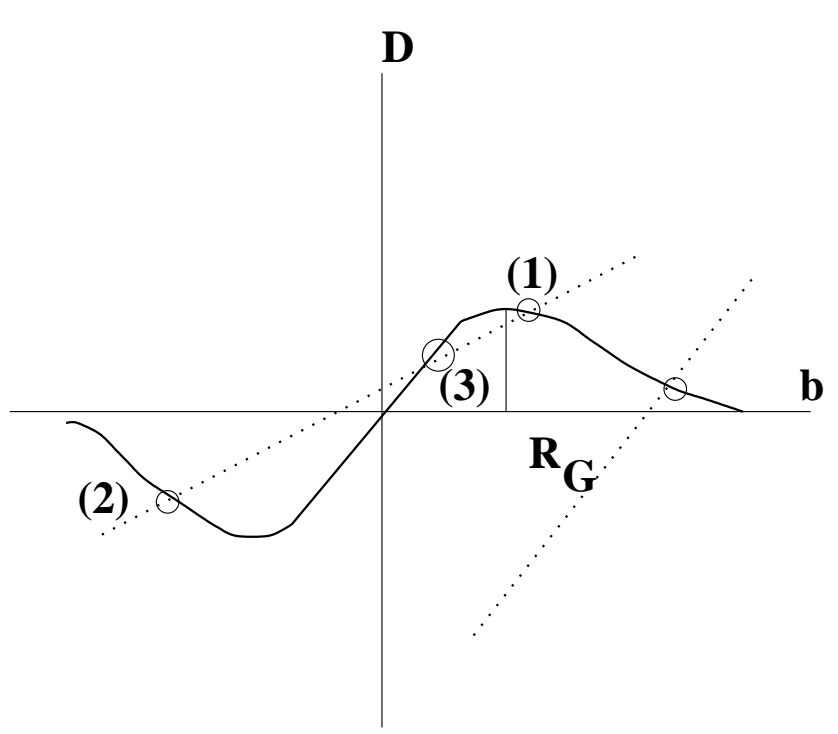

(b)

Figure 3 


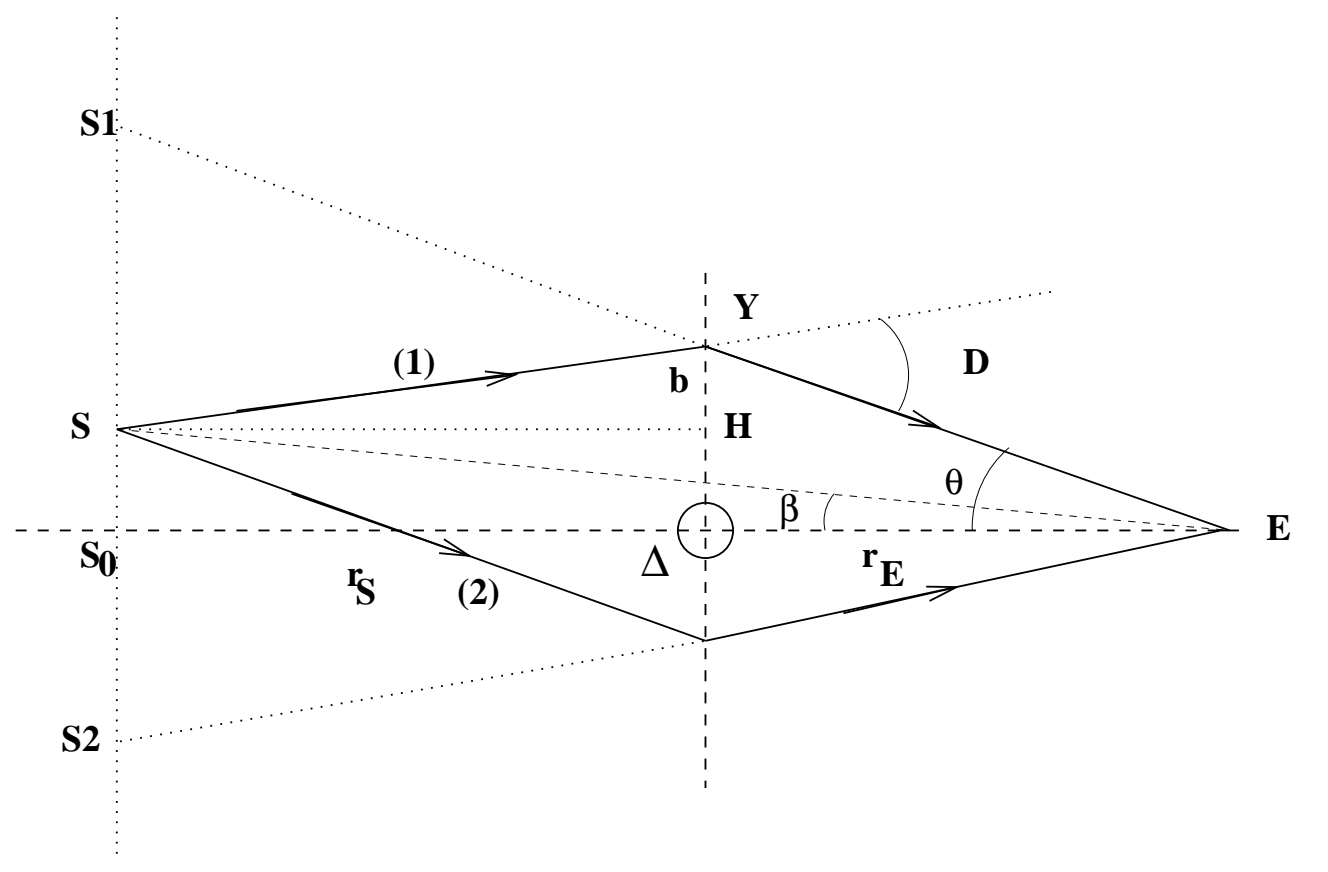

Figure 4 